\title{
Different Types of Scaling in the Dynamics of Period-Doubling Maps Under External Periodic Driving
}

\author{
N. YU. IVAN'KOV' ${ }^{\mathrm{a}, *}$ and S. P. KUZNETSOV ${ }^{\mathrm{b}}$ \\ ${ }^{a}$ College of Applied Sciences, Saratov State University, Astrakhanskaja 83, Saratov, 410026, RF; \\ ${ }^{\mathrm{b}}$ Institute of Radio-Engineering and Electronics RAS, Saratov Division, Zelenaya 38, Saratov, 410019, RF
}

(Received 15 March 2000)

\begin{abstract}
Based on the renormalization group approach developed by Kuznetsov and Pikovsky (Phys. Lett., A140, 1989, 166) several types of scaling are discussed, which can be observed in a neighborhood of Feigenbaum's critical point at small amplitudes of the driving. The type of scaling behavior depends on a structure of binary representation of the frequency parameter: $F$-scaling (Feigenbaum's) for finite binary fractions, $P$ - and $Q$-scaling (periodic and quasiperiodic) for periodic binary fractions, and $S$-scaling (statistical) for non-periodic binary fractions. All types of scaling are illustrated by parameter-plane diagrams for the rescaled Lyapunov exponent.
\end{abstract}

Keywords: Logistic map; Periodic driving; Universality; Scaling

\section{INTRODUCTION}

It is known that many dissipative nonlinear systems of different nature demonstrate the onset of chaos via a period-doubling bifurcation cascade. According to the concept of universality suggested by Feigenbaum [1,2], details of this behavior for the whole class of systems may be described quantitatively using the simplest representative of the class, say, the logistic map.

From the universality intrinsic to an individual system one can conclude that complex constructions, which use such systems as building blocks, should demonstrate universal properties too. Moreover, they could be studied with the use of models built on the basis of the logistic maps.

As a particular example, let us turn to perioddoubling systems driven by external periodic force. According to the above discussion, one can use the forced logistic map to study many aspects of dynamics near the onset of chaos in these systems. The logistic map contains a unique control parameter, so, to account for the external force

\footnotetext{
*Corresponding author.
} 
we introduce a modulation of this parameter and write

$$
x_{n+1}=\lambda+\varepsilon \cos (2 \pi n w+\phi)-x_{n}^{2} .
$$

Here $\varepsilon, w$, and $\phi$ designate, respectively, the amplitude, frequency, and initial phase of the external force. Index $n$ is interpreted as discrete time.

The periodically forced logistic map was studied extensively by many authors [3-9]. There are two essentially different cases: the first, when the frequency parameter $w$ is rational, i.e., a period of the forcing contains an integer number of time steps, and the second, when $w$ is irrational, and one should speak about quasiperiodic rather than periodic driving. In the last case torus doubling bifurcations occur instead of the usual perioddoubling $[3,4,10-12]$, and a possibility of such nontrivial behavior as strange nonchaotic attractor in the intermediate region between order and chaos has been reported $[13,12]$.

Universal properties, which should be regarded as a generalization of those of Feigenbaum, can be expected for small amplitudes $\varepsilon$ and for values of $\lambda$ close to the accumulation point of the period doubling bifurcations in the unforced system, $\lambda_{c} \cong 1.40115518902$. We will refer to the point $\left(\lambda=\lambda_{c}, \varepsilon_{c}=0\right)$ as the Feigenbaum critical point. Renormalization group (RG) analysis appropriate for a neighborhood of this point in the presence of driving was discussed by Arneodo [6], Argoul et al . [7], Kuznetsov [8] and Kuznetsov and Pikovsky [9].

In this paper the scaling properties of the parameter space for the forced logistic map near the Feigenbaum critical point are studied. We closely follow the RG analysis of Ref. [9] with a few modifications. To illustrate scaling we use parameter-plane charts of Lyapunov exponent distributions. We present a sketch of classification for possible types of scaling in dependence on the mathematical nature of the frequency parameter $w$. The simplest one is the usual Feigenbaum scaling ( $F$-scaling), observed for $w$ being equal to a multiple of $2^{-k}$. For other rational values of $w$ the structures in the parameter space near the critical point either reproduce themselves in smaller scales after several steps of doubling ( $P$-scaling), or behave quasiperiodically ( $Q$-scaling). If the frequency parameter $w$ is a typical irrational number, the parameter space structure at subsequent levels of magnification evolves chaotically. However, such a structure may be characterized by some statistically averaged scaling constant ( $S$-scaling).

In continuous-time autonomous perioddoubling systems the dynamics under external driving is more complicated because of presence of additional variable, the phase measured along the trajectory. This class of systems has to be studied separately. Due to the universality following from the RG arguments, the same regularities are expected for other systems - maps and non-autonomous (time-periodic) flow systems.

In Section 2 we recall content of the RG analysis of the forced one-dimensional maps. In Section 3 we discuss and illustrate types of scaling, which occur for rational values of the frequency parameter. In Section 4 statistical scaling intrinsic to a case of irrational frequency is considered. Section 5 contains conclusions and general discussion.

\section{RENORMALIZATION GROUP ANALYSIS}

Idea of the RG approach consists in considering a sequence of evolution operators, corresponding to larger and larger time intervals, each of which is expressed via one or more previous operators. Constructing each next operator in this sequence we use redefined scale (scales) of dynamical variable (variables) to ensure somewhat reasonable limit behavior of the operator sequence. Usually, this is the case at the border to chaos, and we say that the values of parameters of the original system correspond to a kind of critical point. 
Let us write the one-dimensional forced map in a form

$$
\begin{aligned}
x_{n+1}= & f\left(x_{n}\right)+\Delta \lambda \cdot h\left(x_{n}\right) \\
& +\left[\varepsilon Z_{0}\left(x_{n}\right) \exp (i w n+i \phi)+\text { c.c. }\right],
\end{aligned}
$$

where $c . c$. stands for the complex conjugate. Here $f(x), h_{0}(x)$, and $Z_{0}(x)$ are smooth functions. $f(x)$ is supposed to correspond to the critical point of the period-doubling accumulation, while the term $\Delta \lambda \cdot h_{0}(x)$ describes deviation from the criticality. The map (1) is, of course, a particular case of (2) with $f(x)+\Delta \lambda \cdot h_{0}(x)=\lambda-x^{2}=\left(\lambda_{c}+\Delta \lambda\right)-x^{2}$, and $Z_{0}(x)=1 / 2$. Let us apply the map (2) twice to express $x_{n+2}$ in terms of $x_{n}$. Parameters $\varepsilon$ and $\gamma$ are regarded as small, so we consider only linear terms. Then the result will be

$$
\begin{aligned}
x_{n+2}= & f\left(f\left(x_{n}\right)\right) \\
+ & \Delta \lambda \cdot\left[h_{0}\left(x_{n}\right) f^{\prime}\left(f\left(x_{n}\right)\right)+h_{0}\left(f\left(x_{n}\right)\right)\right] \\
+ & \left\{\varepsilon e ^ { i \phi } \left[Z_{0}\left(x_{n}\right) f^{\prime}\left(f\left(x_{n}\right)\right)\right.\right. \\
& \left.\left.+Z_{0}\left(f\left(x_{n}\right)\right) \exp (i w)\right]+ \text { c.c. }\right\}
\end{aligned}
$$

Following Refs. [1,2], instead of $x$ we introduce a rescaled variable

$$
X_{n}=\alpha x_{2 n},
$$

where $\alpha \cong-2.502907$ is Feigenbaum's constant. Note the redefinition of discrete time, which is of principal importance (index $n$ in the left part of (4) versus $2 n$ in the right part). The result of the variable change may be rewritten in a form analogous to (2), but with new functions $f_{1}, Z_{1}$, and new frequency parameter $w_{1}$ :

$$
\begin{aligned}
X_{n+1}= & f_{1}\left(X_{n}\right)+\Delta \lambda \cdot h_{1}\left(X_{n}\right) \\
& +\left[\varepsilon Z_{1}\left(X_{n}\right) \exp \left(i w_{1} n+i \phi\right)+c . c .\right],
\end{aligned}
$$

where

$$
\begin{aligned}
f_{1}(X) & =\alpha f(f(X / \alpha)) \\
h_{1}(X) & =\alpha\left[h_{0}(X) f^{\prime}(f(X))+h_{0}(f(X))\right], \\
Z_{1}(X) & =\alpha\left[Z_{0}(X) f^{\prime}(f(X))+Z_{0}(f(X)) \exp \left(i w_{1}\right)\right], \\
w_{1} & =2 w(\bmod 1) .
\end{aligned}
$$

The new evolution operator defined by a set $\left\{f_{1}, h_{1}, Z_{1}, w_{1}\right\}$ describes the evolution over a doubled time interval. It is expressed via the previous operator by Eqs. (6), which are just the RG equations. Now the same transformation may be applied to operator (5), and then again and again, to obtain recursively a sequence of the evolution operators $\left\{f_{k}, h_{k}, Z_{k}, w_{k}\right\}$ for time intervals $2^{k}$, $k=2,3, \ldots$.

According to Refs. [1,2], the sequence of functions $f_{k}$ converges to the universal function $g(X)$ obeying the functional equation

$$
g(X)=\alpha g(g(X / \alpha)) .
$$

The sequence of $h_{k}$ asymptotically behaves as $\delta^{k} h(X)$, where $h(X)$ is an eigenfunction, and $\delta \cong 4.669201$ is an eigenvalue obtained from the equation

$$
\delta h(X)=\alpha\left[h(X) g^{\prime}(g(X))+h(g(X))\right] .
$$

Both Eqs. (7) and (8) were derived and solved by Feigenbaum, and they correspond to the part of the problem that relates to the unforced system.

For the forced map, we can write a set of equations describing the asymptotic behavior of the evolution operators under subsequent $\mathrm{RG}$ transformations as follows:

$$
\begin{aligned}
X_{n+1}= & g\left(X_{n}\right)+\Delta \lambda \cdot \delta^{k} \cdot h\left(X_{n}\right) \\
& +\left[\varepsilon Z_{k}\left(X_{n}\right) \exp \left(i w_{k} n+\phi\right) c . c .\right], \\
Z_{k+1}(X)= & \alpha\left[Z_{k}(X) g\left(g^{\prime}(X)\right)+Z_{k}(g(X))\right. \\
& \left.\exp \left(i w_{k}\right)\right], \\
w_{k+1}= & 2 w_{k}(\bmod 1) .
\end{aligned}
$$

Observe that the equation for $Z$, in contrast to the equation for $h$, is not invariant with respect to a shift of the index $k$ ("the renormalization time"): This is a functional equation with a variable coefficient $\exp \left(i w_{k}\right)$. The dependence of this coefficient on the renormalization time appears due to evolution of the renormalized frequency parameter $W_{k}$ according to the third Eq. (6), which is the wellknown Bernoulli shift map. Hence, the character of 
this evolution is determined by the binary representation of the initial condition $w=w_{0}$.

If $w_{0}$ is rational, then it is either a finite binary fraction, or a fraction having infinite periodic tail.

If the binary code is finite and contains $m$ digits, then after $m$ steps of the RG transformation we arrive to the fixed point $w_{k} \equiv 0$. At subsequent steps the equation for $Z$ accepts the same form as the equation for $h$, and asymptotic behavior of the solution will be $Z_{k} \propto \delta^{k} h(X)$. So, the influence of small periodic force will be equivalent simply to a shift of the control parameter $\lambda$ from the critical point.

In the case of $w$ represented by an infinite binary fraction of period $m$, the dependence of the coefficient in the equation will be periodic too. Then, like in Floquet theory, one can consider eigenvalues and eigenfunctions defined over the period. This means that they should correspond to multiple RG transformation, containing $m$ steps of the doubling procedure (6).

At last, if $w$ is a typical irrational number, the binary representation will be a random sequence of symbols 0 and 1 . In such a case there is no possibility to express the asymptotic solution simply in terms of eigenfunctions and eigenvalues, but we can introduce an analog of the Lyapunov exponent, which is an averaged characteristic of growth for the solution $Z_{k}$.

\section{RATIONAL FREQUENCIES: SCALING OF $F, P$, AND $Q$ TYPE}

Let us discuss in more detail the scaling properties of the parameter space of the forced logistic map in the case of a rational frequency parameter $w$.

As we have already explained, the case of a finite binary fraction is rather trivial: After some number of steps of the RG procedure the asymptotic behavior of the components $h$ and $Z$ will be identical (up to a constant coefficient), so the evolution operator looks like

$$
X_{n+1} \cong g\left(X_{n}\right)+(\Delta \lambda+c \varepsilon) \cdot \delta^{k} h\left(X_{n}\right) .
$$

The form of this expression depends only on a combination $C=(\Delta \lambda+c \varepsilon) \delta^{\mathrm{k}}$. If we take a special value of $C$, corresponding to a period-doubling bifurcation, then in the $k$-th evolution operator (10) the bifurcation will occur at $(\Delta \lambda+c \varepsilon)=C \delta^{-k}$. This yields a set of bifurcation lines in the parameter plane $(\lambda, \varepsilon)$ accumulated to the Feigenbaum critical line $(\Delta \lambda+c \varepsilon)=0$.

Arrangement of the parameter plane typical for such a case is illustrated in Figure 1a by a diagram for the Lyapunov exponent $\Lambda=\Lambda(\lambda, \varepsilon)$. The value of $\Lambda$ has been computed at each pixel and coded by gray tones. Only regions corresponding to the first period doublings are of somewhat complex form. In smaller vicinity of the critical point the lines of period-doubling bifurcations are parallel and exhibit convergence to the limit critical line according to the usual Feigenbaum's law. This is what we call scaling of $F$ type-a typical picture of Feigenbaum's codimension 1 transition embedded into the two-parameter space.

Now we come to the next, more interesting situation, when the frequency $w$, although rational, is represented by an infinite binary fraction. Due to the rationality, the binary fraction must contain a periodic tail of some period $m$. In this case the dynamics of the renormalized frequencies $w_{k}$ after a number of initial iterations of the RG transformation also becomes periodic, with the same period $m$. To exploit this periodicity we consider the $m$-fold RG transformation (6): This means that instead of the usual doubling in a course of the procedure we increase the original time interval by a factor $2^{m}$. Under subsequent application of this transformation the evolution operators will behave as

$$
\begin{aligned}
X_{n+1}= & g\left(X_{n}\right)+\Delta \lambda \cdot \delta^{m k} h\left(X_{n}\right) \\
& +\left[\varepsilon v^{k} Z_{0}\left(X_{n}\right) \exp \left(i w_{*} n+i \phi\right)+c . c .\right],
\end{aligned}
$$

where the eigenfunction $Z_{0}(X)$, eigenvalue $v$, and constant $w_{*}$ obey the set of equations

$$
\begin{aligned}
Z_{m}(X) & =v Z_{0}(X), \quad w_{0}=w_{m}=w_{*}, \\
Z_{k+1}(X) & =\alpha\left[Z_{k}(X) g\left(g^{\prime}(X)\right)+Z_{k}(g(X)) \exp \left(i w_{k}\right)\right], \\
w_{k+1} & =2 w_{k}(\bmod 1), \quad k=0,1,2, \ldots, m-1 .
\end{aligned}
$$



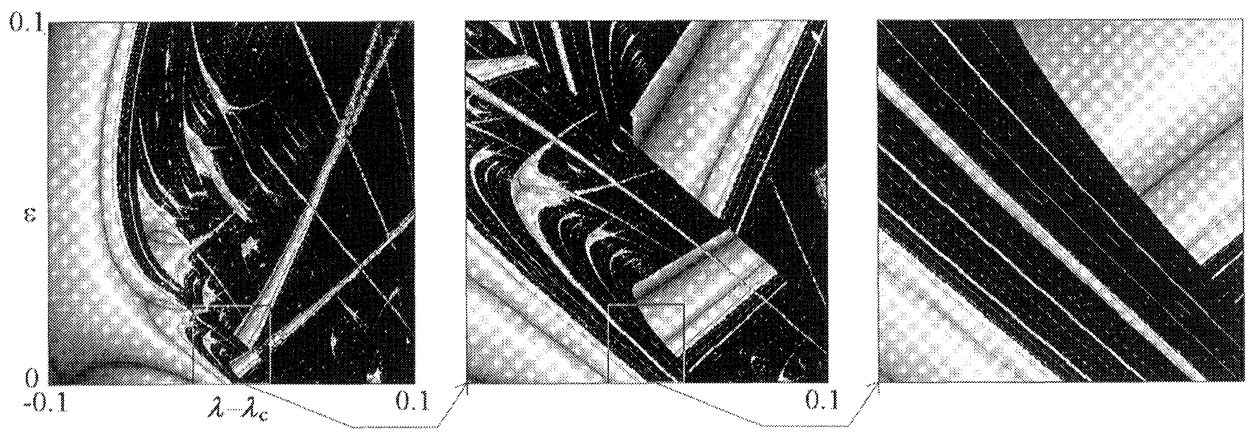

(a)
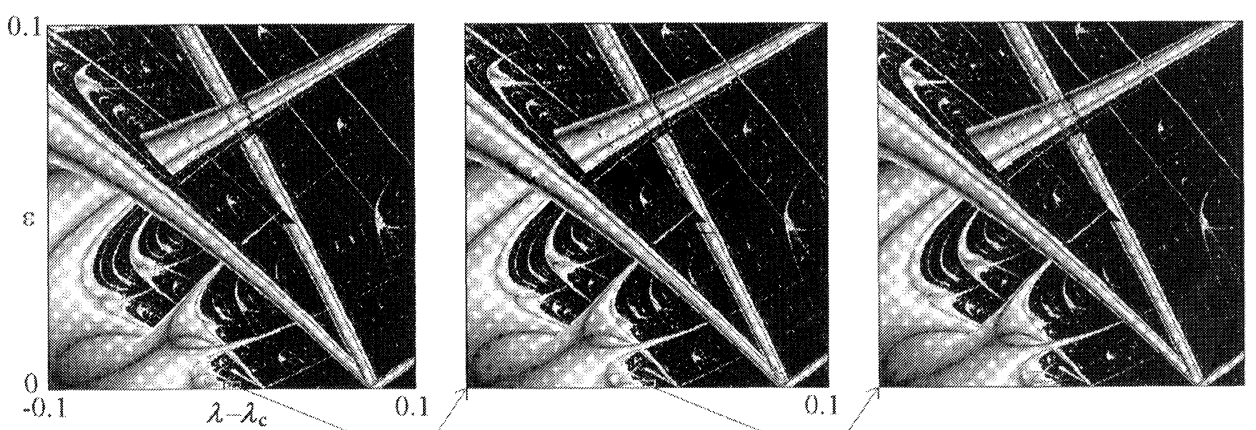

(b)
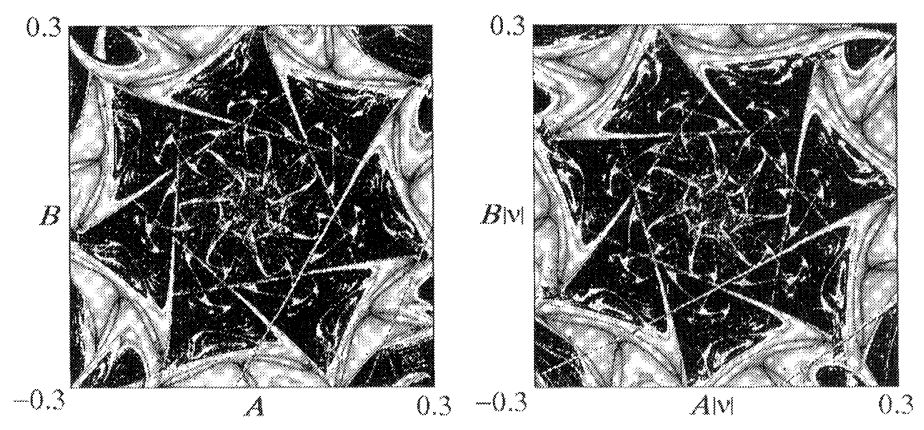

(c)

FIGURE 1 Charts of the Lyapunov exponent distributions on the parameter plane $(\varepsilon, \lambda)$ of the periodically driven logistic map (1) for $w=1 / 8$ (a), $w=1 / 3$ (b), and on the plane $(A=\varepsilon \cos \phi, B=\varepsilon \sin \phi)$ at $\lambda=\lambda_{c}$ for $w=1 / 7$ (c). Gray tones from dark to white correspond to Lyapunov exponent values $\Lambda$ from minus infinity to zero, and black designates positive $\Lambda$ (chaos). Enlargement of small fragments of the pictures illustrates scaling of $F$-type (a), $P$-type (b) and $Q$-type (c). For diagram (a) factors of the enlargement along both coordinate axes are equal to the Feigenbaum constant $\delta \cong 4.6692$; for diagram (b) they are $\delta^{2}$ and $v(1 / 3) \cong 58.96$, respectively, along the horizontal and vertical axes; for diagram (c) the factors are $|v(1 / 7)| \cong 296.27$. The magnification is accompanied by a redefinition of the gray-scale coding rule to outline self-similarity of the Lyapunov exponent plots.

The eigenvalues can be computed from these equations in a straightforward way. Namely, being given a high precision, finite-order polynomial representation of the Feigenbaum universal function $g(X)=1-1.527633 x^{2}+0.104815 x^{4}+$. . . (see $[1,2])$, we construct an analogous representation for functions $Z$, initially with arbitrarily coefficients. Then, a computer program operated with sets of the polynomial coefficients is applied to produce multiple subsequent iterations of the Eqs. (12). After a sufficient number of the iterations only a dominant eigenvector survive, and the 
corresponding eigenvalue may be estimated as the ratio $v=Z_{(k+1) m}(0) / Z_{k m}(0)$. In Table I we present data for frequencies $w$ represented by rationals corresponding to levels $0-6$ of the Farey-SternBrocot tree [14]. Only data for $0 \leq w \leq 1 / 2$ are given because the other half of the unit interval is arranged symmetrically.

Observe that for some values of $w$ the eigenvalues appear to be real; for others they are complex. In these two situations we will discuss scaling properties of the parameter plane separately.

Let us turn first to the case of real eigenvalue $v$; in particular, this is true for $w=1 / 3$ and $1 / 5$. Recall that $m$ designates a period of the binary fraction $w$. The evolution operator defined for $2^{k m}$ iterations of the original map is represented asymptotically by Eq. (11) and depends on the combinations $\Delta \lambda$. $\delta^{m k}$ and $\varepsilon v^{k}$. So, given some definite dynamical regime at $\Delta \lambda$ and $\varepsilon$ we will observe a regime of the same nature at the point $\left(\Delta \lambda / \delta^{m}, \varepsilon / v\right)$, but with the characteristic time scale multiplied by $2^{m}$. All quantitative characteristics of the dynamics for both regimes are expressed one via another in more or less trivial manner. Particularly, the Lyapunov exponent at the second point will differ by the factor $2^{-m}$.

We conclude that the small-scale arrangement of a neighborhood of the critical point exhibits self-similarity. Suppose we have a picture of the parameter plane and increase the resolution step

TABLE I Eigenvalues of Eq. (12) and corresponding RGLE in dependence on the frequency parameter $w$

\begin{tabular}{|c|c|c|c|c|c|}
\hline$w$ & $w$, binary fraction & $|v|$ & $\arg v$ & $\mathrm{~L}$ & Scaling \\
\hline 0 & $0 .(0)$ & 4.669201 & 0 & 1.54098 & $\mathrm{~F}$ \\
\hline $1 / 7$ & $0 .(001)$ & 296.2785 & -0.549255 & 1.89710 & Q \\
\hline $1 / 6$ & $0.0(01)$ & 58.96056 & 0 & 2.03843 & $\mathbf{P}$ \\
\hline $2 / 11$ & $0.0(1011101000)$ & $1.28748 \cdot 10^{8}$ & 0 & 1.86734 & $\mathrm{P}$ \\
\hline $1 / 5$ & $0 .(0011)$ & 2306.061 & 0 & 1.93582 & $\mathrm{P}$ \\
\hline $3 / 14$ & $0.0(011)$ & 296.2785 & 0.549255 & 1.89710 & Q \\
\hline $2 / 9$ & $0 .(001110)$ & 56319.15 & 0 & 1.82313 & $\mathrm{P}$ \\
\hline $3 / 13$ & $0 .(001110110001)$ & $4.96957 \cdot 10^{9}$ & 0 & 1.86055 & $\mathbf{P}$ \\
\hline $1 / 4$ & 0.01 & 4.669201 & 0 & 1.54099 & $\mathrm{~F}$ \\
\hline $4 / 15$ & $0 .(0100)$ & 1195.90 & -0.74177 & 1.77166 & $\mathrm{Q}$ \\
\hline $3 / 11$ & $0 .(0100010111)$ & $1.28748 \cdot 10^{8}$ & 0 & 1.86734 & $\mathrm{P}$ \\
\hline $5 / 18$ & $0.0(100011)$ & 56319.15 & 0 & 1.82313 & $\mathrm{P}$ \\
\hline $2 / 7$ & $0 .(010)$ & 296.279 & -0.549255 & 1.89710 & $\mathrm{Q}$ \\
\hline $5 / 17$ & $0 .(01001011)$ & 6667570 & 0 & 1.96410 & $\mathrm{P}$ \\
\hline $3 / 10$ & $0.0(1001)$ & 2306.06 & 0 & 1.93582 & $\mathrm{P}$ \\
\hline $4 / 13$ & $0 .(010011101100)$ & $4.96957 \cdot 10^{9}$ & 0 & 1.86055 & $\mathrm{P}$ \\
\hline $1 / 3$ & $0 .(01)$ & 58.96056 & 0 & 2.03843 & $\mathrm{P}$ \\
\hline $5 / 14$ & $0.0(101)$ & 296.279 & 0.549255 & 1.89710 & $\mathrm{Q}$ \\
\hline $4 / 11$ & $0.0(1011101000)$ & $1.28748 \cdot 10^{8}$ & 0 & 1.86734 & $\mathrm{P}$ \\
\hline $7 / 19$ & $0.0(101111001010000110)$ & $2.97689 \cdot 10^{14}$ & 0 & 1.85150 & $\mathrm{P}$ \\
\hline $3 / 8$ & 0.011 & 4.669201 & 0 & 1.54099 & $\mathrm{~F}$ \\
\hline $8 / 21$ & $0 .(011000)$ & 47425.2 & -0.442733 & 1.79449 & $\mathrm{Q}$ \\
\hline $5 / 13$ & $0.0(110001001110)$ & $4.96957 \cdot 10^{9}$ & 0 & 1.86055 & $\mathrm{P}$ \\
\hline $7 / 18$ & $0 .(011000)$ & 56319.15 & 0 & 1.82313 & $\mathbf{P}$ \\
\hline $2 / 5$ & $0 .(0110)$ & 2306.061 & 0 & 1.93582 & $\mathrm{P}$ \\
\hline $7 / 17$ & $0.0(11010010)$ & 6667570 & 0 & 1.96410 & $\mathrm{P}$ \\
\hline $5 / 12$ & $0.01(10)$ & 58.96056 & 0 & 2.03843 & $\mathrm{P}$ \\
\hline $8 / 19$ & $0 .(011010111100101000)$ & $2.97689 \cdot 10^{14}$ & 0 & 1.85150 & $\mathrm{P}$ \\
\hline $3 / 7$ & $0 .(011)$ & 296.279 & 0.549255 & 1.89710 & $\mathrm{Q}$ \\
\hline $7 / 16$ & 0.111 & 4.669201 & 0 & 1.54099 & $\mathrm{~F}$ \\
\hline $4 / 9$ & $0 .(011100)$ & 56319.15 & 0 & 1.82313 & $\mathrm{P}$ \\
\hline $5 / 11$ & $0.0(1110100010)$ & $1.28748 \cdot 10^{8}$ & 0 & 1.86734 & $\mathrm{P}$ \\
\hline $1 / 2$ & 0.1 & 4.669201 & 0 & 1.54099 & $\mathrm{~F}$ \\
\hline
\end{tabular}


by step, each step revealing the structure associated with the next time-scale doubling. Then the observed picture will repeat itself after each $m$ step of the procedure.

This type of behavior we call P-scaling and illustrate it in Figure $1 \mathrm{~b}$. Note the reproduction of the structure under enlargement by a factor $\delta^{m}$ along horizontal axis and by $v$ along the vertical one. (If the periodic tail of the binary representation of $w$ starts from the $M$-th digit, this self-similarity is revealed only in sufficiently small scales: deflection from the critical point $\Delta \lambda$ should be of order $\delta^{-M}$ or less.)

We must add now that the configuration in the parameter plane $(\lambda, \varepsilon)$ depends also on the phase constant $\phi$. However, this dependence remains the same at all levels of the small-scale resolution because the eigenvalue $v$ is real.

When the property of scaling is stated, we can use it to reconstruct the parameter space structure near the critical point on an arbitrarily large number of levels of resolution.

Let us turn to diagrams of the parameter plane $(\Delta \lambda, \varepsilon)$ in double logarithmic scale. In such a plot the parameter change $\Delta \lambda \longrightarrow \Delta \lambda / \delta^{m}, \varepsilon \longrightarrow \varepsilon / v$ corresponds to a shift along the two coordinate axes by $m \ln \delta$ and $\ln v$, respectively. Accounting scaling, we may perform the computations for one definite interval of $\Delta \lambda$ for a set of $w$ values visited in a course of the RG transformation, and then to arrange the pictures in the double logarithmic plot to cover the desirable range of $\lambda$ and $\varepsilon$. In Figure 2 we present such collages. Now the gray tones code the Lyapunov exponent normalized by factor $|\Delta \lambda|^{\gamma}$, where $\gamma=\log _{\delta} 2 \cong 0.4498$. This rule is selected on a basis of the well known scaling relation for the Lyapunov exponent near the Feigenbaum's critical point $[1,2,6,7]$. It ensures uniformity of the gray scale coding in the whole range of parameters including arbitrarily small vicinity below the critical value of $\lambda$. Compare simple form of the chaos border in a case of $F$ scaling (a) with periodic structure of the border characteristic for $P$-scaling (b).
Another type of small-scale parameter plane structure near the Feigenbaum critical point arises when the eigenvalue $v$ is complex, for example, at $w=1 / 7$ or $1 / 15$. In such case one should rewrite Eq. (11) as

$$
\begin{aligned}
X_{n+1}= & g\left(X_{n}\right)+\Delta \lambda \delta^{m k} h\left(X_{n}\right) \\
& +\left\lfloor\varepsilon|v|^{k} Z_{0}\left(X_{n}\right) \exp \left(i w_{*} n+i \phi_{k}\right)+c . c .\right\rfloor, \\
\phi_{k}= & \phi+k \arg v .
\end{aligned}
$$

The numerically calculated arguments of the complex eigenvalues are found not to be in rational ratios with $2 \pi$. This means that the value of phase $\phi_{k}$ is different at each new step $k$. Hence, there is no exact repetition of the evolution operators, and, consequently, no exact reproduction of forms in the parameter plane under subsequent enlargement in the neighborhood of the critical point. These forms behave quasiperiodically, and we call it $Q$-scaling. To give evidence of absence of the periodicity we present in Figure 2c a collage in double logarithmic scale. It is constructed from a set of plots computed separately in a similar way as it has been done above in the context of $P$-scaling.

In fact, what is observed is a two-dimensional cross-section of a higher-dimensional periodic structure. Indeed, let us consider the extended three-dimensional parameter space $(\lambda, A, B)$, where $A=\varepsilon \cos \phi, B=\varepsilon \sin \phi$. Let us have some dynamical regime for given $\Delta \lambda, A$ and $B$. Now take another point in the parameter space, namely, $\left(\Delta \lambda / \delta^{m}, A^{\prime}\right.$, $B^{\prime}$ ), where $A^{\prime}$ and $B^{\prime}$ are defined via $A^{\prime}+i B^{\prime}=$ $(A+i B) / v$. The scale change of $\Delta \lambda$ is accompanied by a rotation by an angle $(-\arg v)$ in $(A, B)$-plane. Then the form of the evolution operator at the $(k+1)$-th step coincides with that at the old point at the $k$-th step, and, so, the regimes at both the points will be of the same nature. In Figure 1c we present an illustration. A cross section of the three-dimensional parameter space by the plane $\lambda=\lambda_{c}$ is shown with gray-scale coding of the Lyapunov exponent distribution. Observe that two pictures representing the structure in 


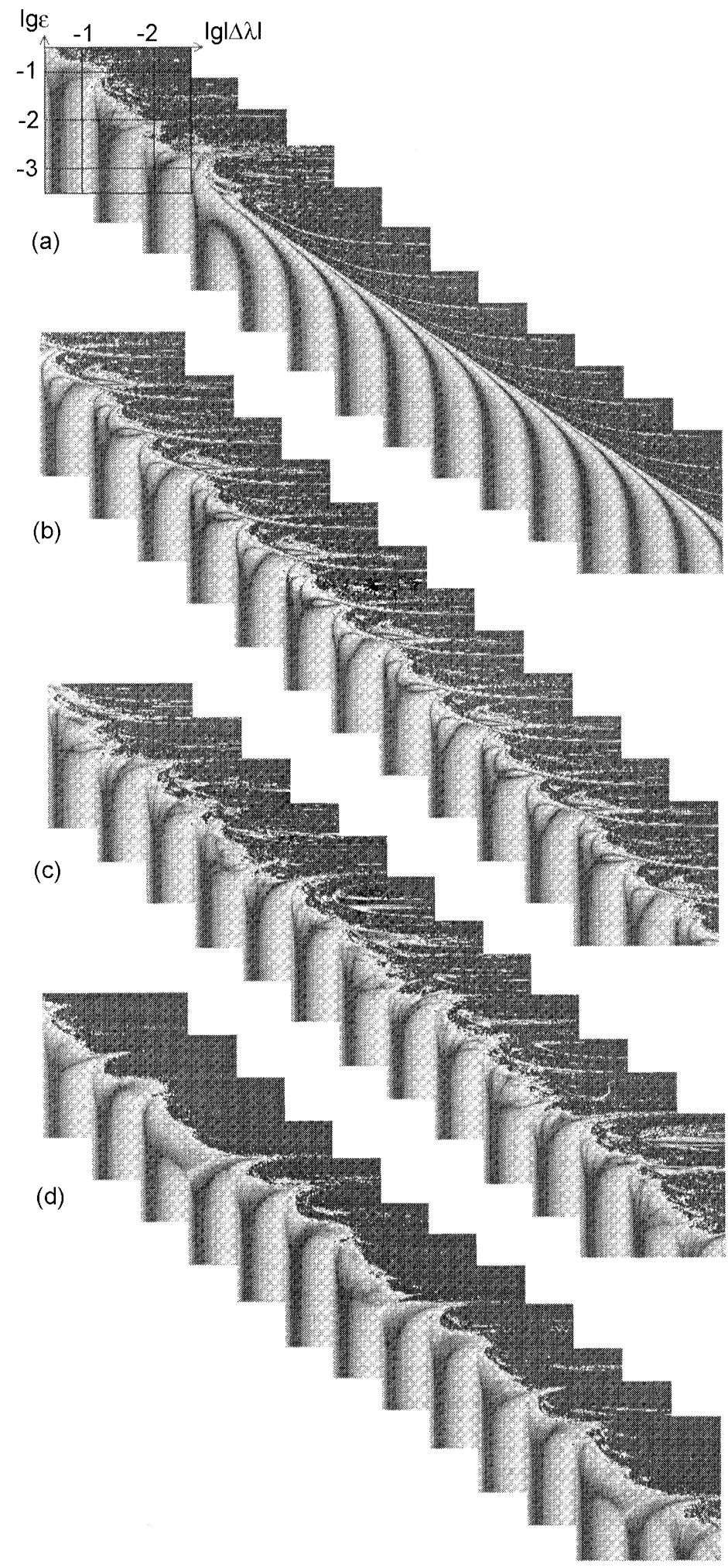

FIGURE 2 Collages built from sequences of plots in double logarithmic scale $\left(\lg \left|\lambda-\lambda_{c}\right|, \lg \varepsilon\right)$. Gray tones from dark to white designate values of the rescaled Lyapunov exponent $\Lambda /\left|\lambda-\lambda_{c}\right|^{n}, \gamma \cong 0.4498$, from minus infinity to zero, black designates positive $\Lambda$ (chaos). Observe the simple self-similarity of the picture at small scales in the case of $F$-scaling, $w=1 / 8$ (a), periodic repetition of the forms $-P$-scaling, $w=1 / 3$ (b), quasiperiodic evolution of the forms - $Q$-scaling, $w=1 / 7$ (c), and chaotic evolution for randomly chosen $w$ (see (18)) $-S$-scaling (d). 
different scales coincide up to rotation through a definite angle.

\section{RENORMALIZATION GROUP LYAPUNOV EXPONENT, IRRATIONAL FREQUENCIES AND CHAOTIC SCALING}

Now we are going to consider the case of irrational frequency parameter. Let us suppose that it is chosen by chance on the unit interval. With probability 1 , the binary representation for such a number will be a random sequence of symbols 0 and 1. Subsequent iterations of the Bernoulli map for the frequency parameter will be chaotic, so, the relation for $Z_{k}$ (see (12)) becomes a functional equation with random coefficient. Nevertheless, in average, the solution will grow exponentially in dependence on the renormalization time $k$. We may introduce a quantitative characteristic of this growth as

$$
L=\varlimsup_{k \rightarrow \infty} \frac{1}{k} \ln |Z(0)|
$$

This reminds the definition of the Lyapunov exponent, and we call this value the renormalization group Lyapunov exponent (RGLE). (It should not be confused with usual Lyapunov exponent, which we designate $\Lambda$.)

To reveal the meaning of the RGLE we note that under subsequent iterations of the RG transformation the term proportional to $\varepsilon$ in the evolution operator grows, in average, as $e^{k L}$, while the term proportional to $\Delta \lambda$ grows as $\delta^{k}$. If we wish to realize a dynamical regime approximately similar to the original one, we have to rescale $\Delta \lambda$ as $\delta^{-k}$ and $\varepsilon$ as $e^{-k L}$. In the double logarithmic scale it corresponds to a shift along two coordinate axes by $k \ln \delta$ and $k L$, respectively. Approximately similar regimes will be placed, hence, along the straight line of the slope $L / \ln \delta$. In particular, the border of chaos, which is of rather complicated form, goes in average along such a straight line.
Obviously, the RGLE may be defined for rational frequencies too. In particular, for the case of $F$-scaling, we have simply

$$
L=\ln \delta=1.54098 \ldots
$$

In the cases of $P$ - and $Q$-scaling the RGLE is easily found to be

$$
L=\ln |\nu| / m,
$$

where $v$ is the dominant eigenvalue, and $m$ is the period of the periodic tail for the binary fraction $w$. In Table I, besides the eigenvalues $v$, we have presented also the corresponding RGLE. Note that they differ one from another not so much as the eigenvalues. From Figure 2 one can see that the border of chaos indeed follows approximately the line of slope $L / \ln \delta$.

For a typical irrational $w$, randomly chosen from the unit interval, the value of $L$ must be a universal constant. According to estimates of Refs. [8,9],

$$
L \cong 1.82 \text { and } \quad L / \ln \delta \cong 1.18
$$

In Figure $2 \mathrm{~d}$ we present a collage similar to those previously discussed, but for irrational frequency parameter chosen as a random binary fraction, namely,

$$
\begin{aligned}
w= & 0.101001111000101111001001100 \\
& 111111100111101 \ldots
\end{aligned}
$$

It may be seen that the forms do not repeat each other, so there is no scaling in usual meaning. However, it is possible yet to speak about approximate repetition and scaling in a weaker, statistical sense ("renormalization chaos"). The border of chaos runs along the straight line of slope 1.18, as predicted.

\section{CONCLUSION}

In this paper we have discussed scaling properties of the parameter space for the forced logistic map 
near Feigenbaum's critical point, which follow from the RG analysis [9]. The character of scaling behavior depends on the mathematical nature of the frequency parameter $w$, namely, on the structure of its binary representation. We distinguish several types of scaling:

- usual Feigenbaum scaling ( $F$-scaling) for $w$ being a multiple of $2^{-k}$;

- periodic scaling ( $P$-scaling), when the structures in the parameter plane reproduce themselves in smaller scales after several steps of the scale change;

- quasiperiodic scaling ( $Q$-scaling), when the twodimensional parameter plane structure appears to be quasiperiodic, although it may be considered as the cross-section of a periodic threedimensional structure;

- statistical scaling ( $S$-scaling), which takes place for typical irrational numbers, when the forms of structures in the parameter plane are reproduced only approximately, being similar in a statistical sense.

As follows from the RG analysis, the forced logistic map may be regarded as a representative of the universality class, which contains numerous period doubling systems under external periodic or quasiperiodic force. All of them are expected to manifest the same regularities as the logistic map. It would be interesting to observe these regularities in numerical and experimental studies on higher dimensional systems.

\section{Acknowledgement}

This work was supported, in part, by Ministry of Education of RF (grant No. 97-0-8.3-88).

\section{References}

[1] Feigenbaum, M. (1978). J. Stat. Phys., 19, 25.

[2] Feigenbaum, M. (1979). J. Stat. Phys., 21, 669.

[3] Kaneko, K. (1983). Progr. Theor. Phys., 69, 1806.

[4] Kaneko, K. (1984). Progr. Theor. Phys., 72, 202.

[5] Heldstab, J., Thomas, H., Geisel, T. and Radons, G. (1983). Z. Phys., B50, 141.

[6] Arneodo, A. (1984). Phys. Rev. Lett., 53, 1240; 54, 86.

[7] Argoul, F., Arneodo, A., Richetti, O., Roux, J. C. and Swinney, H. L. (1987). Europhys. Lett., 3, 643.

[8] Kuznetsov, S. P. (1984). JETP Lett., 39, 133.

[9] Kuznetsov, S. P. and Pikovsky, A. S. (1989). Phys. Lett., A140, 166.

[10] Arneodo, A., Collet, P. H. and Spiegel, E. A. (1983). Phys. Lett., A94, 1.

[11] Anishchenko, V. S. (1995). Dynamical Chaos-Models and Experiments, World Scientific, Singapore.

[12] Kuznetsov, S., Pikovsky, A. and Feudel, U. (1998). Phys. Rev., E57, 1585.

[13] Grebogi, C., Ott, E., Pelikan, S. and Yorke, J. A. (1984). Physica, D13, 261.

[14] Graham, R. L., Knuth, D. E. and Patashnik, O. (1989). Concrete Mathematics, Addison-Wesley Publ. 


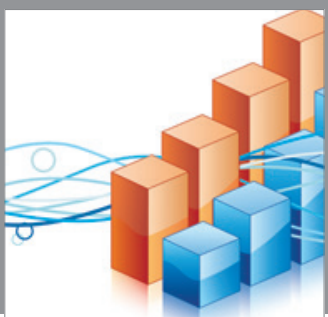

Advances in

Operations Research

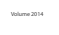

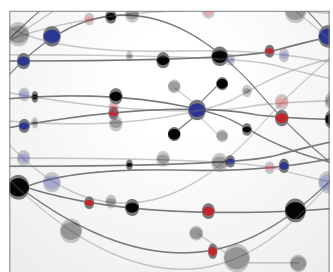

\section{The Scientific} World Journal
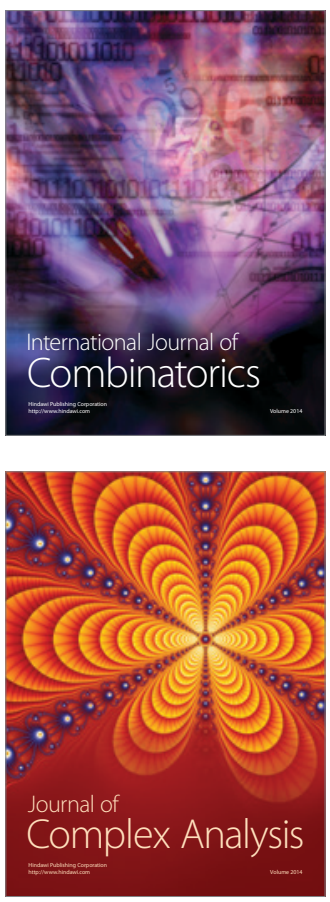

International Journal of

Mathematics and

Mathematical

Sciences
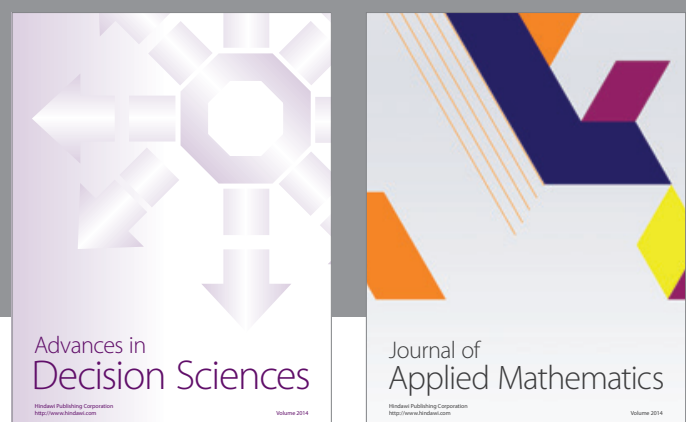

Journal of

Applied Mathematics
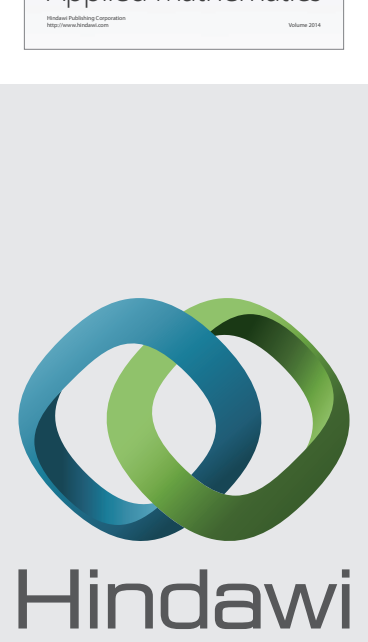

Submit your manuscripts at http://www.hindawi.com
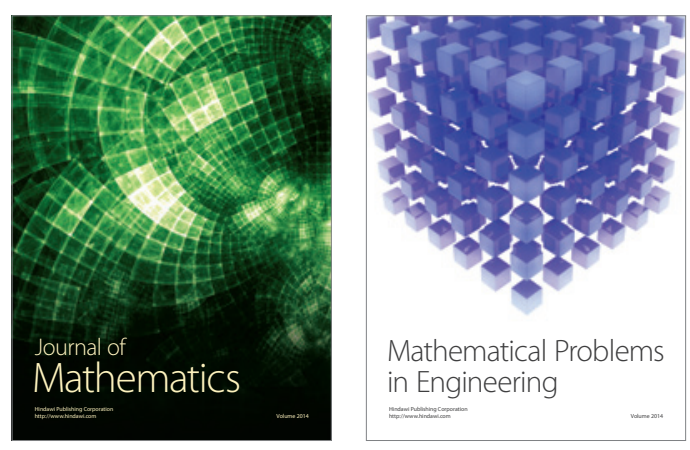

Mathematical Problems in Engineering
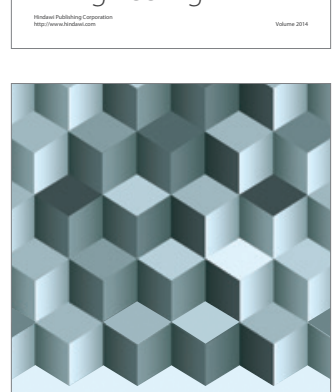

Journal of

Function Spaces
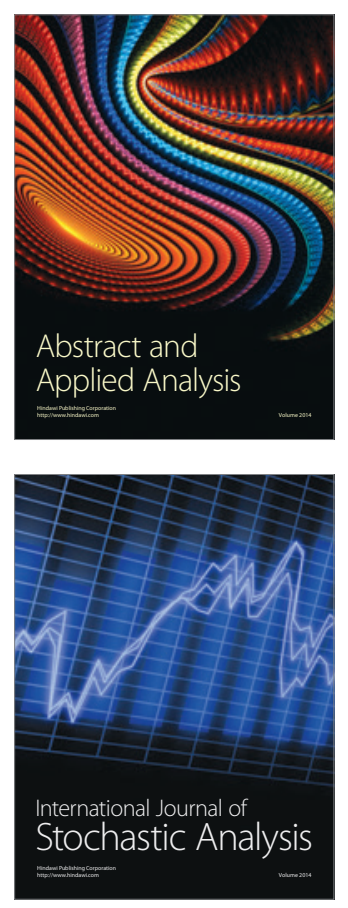

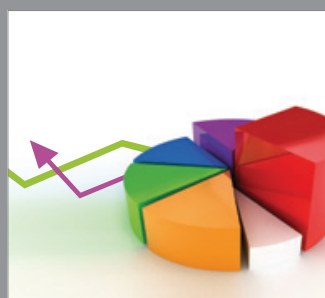

ournal of

Probability and Statistics

Promensencen
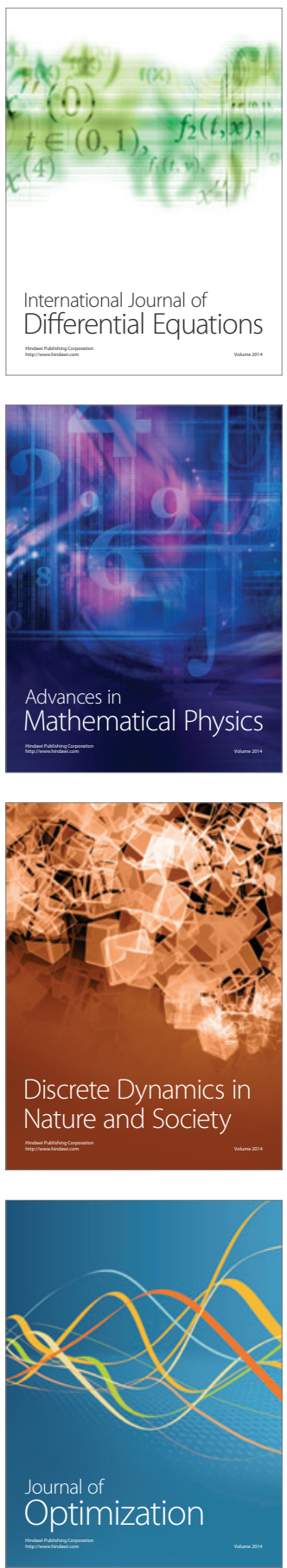